\title{
Les enseignants et le renouveau collégial au Québec : analyse interactionniste de la construction et de la négociation du sens d'un changement de pratique prescrit Teachers and the collegial renewal in Québec: an interactional analysis of the construction and negotiation of the meaning of a change in prescribed practice
} La enseñanza y renovación colegial en Quebec: análisis
interaccionista de la construcción y de la negociación del
significado de un cambio de práctica prescrito

Éliane Dulude et Martial Dembélé

Volume 40, numéro 1, printemps 2012

La gestion et l'appropriation du changement en éducation

URI : https://id.erudit.org/iderudit/1010151ar

DOI : https://doi.org/10.7202/1010151ar

\section{Aller au sommaire du numéro}

\section{Éditeur(s)}

Association canadienne d'éducation de langue française

\section{ISSN}

0849-1089 (imprimé)

1916-8659 (numérique)

\section{Découvrir la revue}

Citer cet article

Dulude, É. \& Dembélé, M. (2012). Les enseignants et le renouveau collégial au Québec : analyse interactionniste de la construction et de la négociation du sens d'un changement de pratique prescrit. Éducation et francophonie, 40(1), 160-175. https://doi.org/10.7202/1010151ar
Résumé de l'article

Le présent article prend appui sur une étude exploratoire visant à comprendre le processus de construction et de négociation du sens d'une réforme par les enseignants d'un département de langues modernes au collégial au Québec. L'article a pour but de mettre en lumière l'influence relative des symboles, du coordonnateur et des interactions sociales dans les changements de pratique. Les données proviennent principalement d'entrevues semi-dirigées avec les enseignants participants, complétées par une analyse de contenu de prescriptions ministérielles. Il ressort de l'analyse des données, entre autres, que l'épreuve synthèse est devenue un objet de prestige dans ce département et exerce une pression constante sur les interactions entre enseignants ainsi que sur les priorités d'action.

Tous droits réservés $@$ Association canadienne d’éducation de langue française, Ce document est protégé par la loi sur le droit d’auteur. L’utilisation des 2012 services d'Érudit (y compris la reproduction) est assujettie à sa politique d'utilisation que vous pouvez consulter en ligne.

https://apropos.erudit.org/fr/usagers/politique-dutilisation/ 


\title{
Les enseignants et le renou- veau collégial au Québec: analyse interactionniste de la construction et de la négocia- tion du sens d'un changement de pratique prescrit
}

\author{
Éliane DULUDE \\ Université de Montréal, Québec, Canada \\ Martial DEMBÉlÉ \\ Université de Montréal, Québec, Canada
}

\section{RÉSUMÉ}

Le présent article prend appui sur une étude exploratoire visant à comprendre le processus de construction et de négociation du sens d'une réforme par les enseignants d'un département de langues modernes au collégial au Québec. L'article a pour but de mettre en lumière l'influence relative des symboles, du coordonnateur et des interactions sociales dans les changements de pratique. Les données proviennent principalement d'entrevues semi-dirigées avec les enseignants participants, complétées par une analyse de contenu de prescriptions ministérielles. Il ressort de l'analyse des données, entre autres, que l'épreuve synthèse est devenue un objet de prestige dans ce département et exerce une pression constante sur les interactions entre enseignants ainsi que sur les priorités d'action. 


\section{ABSTRACT}

\section{Teachers and the collegial renewal in Québec: an interactional analysis of the construction and negotiation of the meaning of a change in prescribed practice}

Éliane DULUDE and Martial DEMBÉLÉ

University of Montréal, Québec, Canada

This article is based on an exploratory study on understanding the process used by teachers in the modern languages department of a Québec college to construct and negotiate the meaning of a reform. The article aims to highlight the relative influence of symbols, the coordinator, and social interactions on changes in practice. The data comes mainly from semi-directed interviews with participating teachers, followed by an analysis of the contents of ministerial specifications. One of the main outcomes of the data analysis is that exams have become an object of prestige in this department and put constant pressure on the interactions between teachers and priorities for action.

\section{RESUMEN}

\section{La enseñanza y renovación colegial en Quebec: análisis interaccionista de la construcción y de la negociación del significado de un cambio de práctica prescrito.}

Éliane DULUDE y Martial DEMBÉLÉ

Universidad de Montreal, Quebec, Canadá

El presente artículo se apoya en un estudio exploratorio que trata de comprender el proceso de construcción y de negociación del significado de la reforma entre los maestros de un departamento de lenguas modernas de nivel colegial en Quebec. El objetivo del artículo es destacar la influencia relativa de los símbolos, del coordinador y de las interacciones sociales en los cambios de práctica. Los datos provienen de entrevistas semi-dirigidas con los maestros participantes, completadas con un análisis de contenido de las prescripciones ministeriales. El análisis de los datos muestra, entre otras cosas, que el examen de síntesis se ha convertido en un objeto de prestigio en dicho departamento y que ejerce una presión constante sobre las interacciones entre los maestros y sobre sus prioridades de acción. 


\section{Contexte général}

En 1964, la commission Parent dépose un rapport faisant état de la situation du système d'éducation au Québec. Le rapport critique, entre autres, les difficultés d'accessibilité aux études supérieures et le faible niveau de scolarité des Québécois (Robert et Tondreau, 1998). Il recommande la création d'un nouvel ordre d'enseignement supérieur (Gingras, 1992a). Celui-ci se nommera collège d'enseignement général et professionnel ou, selon son abréviation commune, CÉGEP. Il a pour mandat d'offrir une formation préparant les étudiants à des études universitaires ou à exercer un métier de technicien de niveau supérieur (Ministère de l'Enseignement supérieur et de la Science, 1993). Ce nouvel ordre d'enseignement vise un certain nombre d'objectifs, dont l'accessibilité pour tous, la démocratisation du système, l'augmentation de la scolarisation, la hausse de la qualification de la main-d'œuvre technique (Ministère de l'Enseignement supérieur et de la Science, 1993). Les activités connexes des cégeps sont, entre autres, la recherche, le transfert de technologie, le développement régional et l'action internationale (Ministère de l'Enseignement supérieur et de la Science, 1993). Les premiers établissements collégiaux sont fondés en 1967 (Dorais, 1992).

Durant les années 1990, certains changements sociaux, économiques et politiques se répercutent sur l'enseignement collégial, soulevant des interrogations par rapport à son rôle dans la société. La réalité sociale a grandement changé au Québec depuis les années 1960 (diversité ethnoculturelle croissante, diversité des modes de fréquentation scolaire ${ }^{1}$, valeurs pluralistes) (Conseil supérieur de l'éducation, 1998). La mondialisation fait émerger de nouvelles normes et des standards internationaux. La notion de compétences est au cœur des préoccupations à l'égard des diplômés puisque leur formation témoigne de leur capacité à concurrencer sur le plan international (Ministère de l'Enseignement supérieur et de la Science, 1993). Sur le plan politique, il semble primordial de consolider et rationaliser l'enseignement supérieur. Cela implique pour les collèges des amendements législatifs et réglementaires en vue d'accroître leur autonomie, d'assouplir l'encadrement administratif, de favoriser la création de collèges régionaux et le regroupement des services (Robert et Tondreau, 1997). Ces amendements sont effectués dans le cadre de ce qui est communément appelé le «renouveau collégial».

\section{Grandes lignes du renouveau collégial}

Introduit en 1993, le renouveau collégial au Québec vise la transformation en profondeur des mécanismes institutionnels, notamment l'organisation des programmes par la mise en œuvre de nouvelles prescriptions (Commission de l'évaluation de l'enseignement collégial, 2001; Dorais, 1992). Quatre cibles stratégiques sont proposées afin d'atteindre les objectifs du renouveau collégial. La première cible

1. Étudiants qui concilient études et travail, jeunes adultes qui retournent aux études. 
stratégique, nommée "nouveau défi d'accessibilité: la réussite des études» (Ministère de l'Enseignement supérieur et de la Science, 1993), vise non seulement une plus grande accessibilité aux études, mais aussi la réussite du plus grand nombre. La seconde cible stratégique concerne les programmes d'études. Elle propose la réorganisation des programmes de manière à ce qu'ils soient plus exigeants, cohérents et adaptés aux besoins des étudiants. L’approche par compétences est également privilégiée. Caractérisée par «des objectifs formulés en fonction de compétences (habiletés, connaissances, attitudes et comportements) à atteindre et des standards liés à ces objectifs au collégial» (MEQ, 2000, p. 11), cette approche vise l'intégration des savoirs par un nouveau mode d'organisation suivant une logique de séquence des cours à partir d'un référentiel commun. La troisième cible stratégique transfère les responsabilités pédagogiques aux établissements d'enseignement et suggère des dispositifs d'évaluation plus rigoureux (Ministère de l'Enseignement supérieur et de la Science, 1993). Par conséquent, les collèges doivent revoir et raffermir leur politique institutionnelle d'évaluation des programmes, en plus d'élaborer une épreuve synthèse destinée aux finissants (Dorais, 1992). Cette épreuve a pour but de déterminer si les étudiants ont atteint les objectifs et les standards fixés par le programme d'études en les plaçant en situation réelle et en évaluant s'ils en ont intégré ou non les objectifs terminaux. La dernière cible stratégique incite à renouveler et à resserrer les liens entre les collèges et leurs partenaires (Dorais, 1992). Les cibles stratégiques 2 et 3 retiennent davantage notre attention, car elles touchent directement l'organisation du travail et la tâche enseignante.

La formation pédagogique des enseignants devient alors une préoccupation urgente pour les milieux collégiaux. Les enseignants ont des formations disciplinaires très diversifiées, peu importe le secteur (préuniversitaire ou technique) auquel ils appartiennent. La considération d'une pédagogie collégiale s'impose donc afin de compléter ces formations par des notions pédagogiques (Gingras, 1992b). Le besoin urgent est de former les enseignants en fonction des caractéristiques particulières du milieu, y compris les caractéristiques de la population étudiante au collégial (composée de jeunes et d'adultes), de clarifier les objectifs et les contenus des programmes préuniversitaires et techniques et de créer du matériel didactique spécifique pour chaque discipline (Gingras, 1992b). Les premières tentatives d'expliciter une pédagogie propre au collégial et de mettre en place un modèle de perfectionnement pédagogique nommé PERFORMA ${ }^{2}$ sont amorcées en collaboration avec les conseillers pédagogiques, la Fédération des cégeps et un comité d'étude (Gingras, 1992b).

2. Un programme de perfectionnement en pédagogie offert dans les collèges par l'Université de Sherbrooke (Québec). 


\section{Difficultés d'implantation du renouveau et implications pour la pratique enseignante}

Différents acteurs ont identifié certains obstacles rencontrés au cours de l'implantation du renouveau collégial. Le Conseil supérieur de l'éducation (1998) estime que l'implantation de cette réforme a été précipitée. Selon le Conseil, les assises théoriques de la réforme n'étaient pas encore clairement définies. L'interprétation des prescriptions ministérielles était donc un défi de taille pour les acteurs des établissements collégiaux (CSE, 2000), ce qui a rendu l'implantation plus ardue. Cette constatation trouve écho dans les travaux de Desimone (2002), qui note que moins les lignes directrices d'une réforme sont précises, plus longue est la période d'implantation. Les assises théoriques n'étant pas bien définies, la tâche d'interprétation par les administrateurs, les coordonnateurs et les enseignants s'est complexifiée.

Certaines prescriptions ministérielles (l'approche-programme, les programmes d'études élaborés par compétences, la décentralisation de certaines tâches administratives) semblent donc plus difficiles à s'approprier pour les acteurs (Conseil supérieur de l'éducation, 1998; 2000). La Fédération des enseignantes et enseignants de cégep (1999) affirme que les prescriptions ministérielles alourdissent, modifient et complexifient la tâche des enseignants au collégial. On fait référence, entre autres, à l'augmentation significative du nombre d'heures de travail nécessaire à la planification des cours (CSE, 1998; FEC, 1999). La Fédération des enseignantes et enseignants de cégep (FEC) identifie un certain nombre de facteurs qui auraient contribué de façon significative à l'alourdissement de leur travail: «la décentralisation des activités d'apprentissage, la formation générale propre au programme, les modalités d'évaluation, l'évaluation des programmes et l'élaboration de cours d'intégration et d'épreuves synthèses» (FEC, 1999). Pour sa part, le comité patronal de négociation des collèges note que des changements sont effectivement apportés par le renouveau et que la pratique enseignante évolue, sans toutefois en préciser le sens (CPNC, 2004). Il ne fait pas mention d'un alourdissement de la tâche ou d'une complexification du travail. Les avis du Conseil supérieur de l'éducation, les rapports syndicaux et patronaux tendent à confirmer que les changements sont toujours en cours, mais qu'ils ne semblent pas faire l'unanimité quant à l'alourdissement ou à la complexification du travail enseignant. C'est pourquoi nous avons choisi de nous limiter aux prescriptions ministérielles évoquées par le Conseil supérieur de l'éducation, la Fédération des enseignantes et enseignants de cégep et le Comité patronal de négociation comme ayant une incidence sur la tâche enseignante.

Les changements paradigmatiques proposés par ces prescriptions ministé rielles sont profonds, car celles-ci exigent de nouveaux modes d'organisation et d'évaluation des programmes et de nouvelles méthodes d'enseignement et d'évaluation pour les enseignants. Or, nous savons que plus le changement est majeur, plus la construction de sens est difficile, car elle requiert une collaboration et une négociation constantes entre les différents acteurs (Spillane, Reiser, et Reimer, 2002). Ce sont, entre autres, le sens à donner aux prescriptions ministérielles et les nouvelles valeurs qu'elles véhiculent qui suggèrent un saut conceptuel important. Parmi les nombreuses 
prescriptions ministérielles, nous avons retenu celles qui nous paraissent les plus complexes, à savoir l'approche-programme, l'approche par compétences, l'évaluation des compétences et l'élaboration de l'épreuve synthèse. Nous pouvons ainsi examiner la manière dont les enseignants construisent du sens et répondent à des prescriptions ministérielles visant le changement de pratique pédagogique. Dans le présent article, nous tentons de mettre en lumière la centralité des symboles, du rôle du coordonnateur et des interactions sociales dans le processus interprétatif des changements prescrits dans la pratique enseignante.

\section{Mode d'investigation et perspective théorique}

La description et la compréhension de cas particuliers sont une exigence de base de l'étude de la construction de sens par des acteurs. L'étude de cas nous est donc apparue comme le mode d'investigation le plus approprié. Ce mode d'investigation est particulariste et permet d'étudier en profondeur des situations ou phénomènes sociaux afin d'en saisir la complexité et la richesse (Karsenti et Demers, 2000; Merriam, 1988). Dans le cas présent, il s'agit de chercher à comprendre le sens qu'un groupe de quatre enseignants vivant des changements dans leur pratique attribue aux prescriptions ministérielles. Ces enseignants sont rattachés au département de langues modernes (espagnol et allemand) d'un établissement collégial situé sur l'île de Montréal. Ils sont d'origine québécoise et hispanique ${ }^{3}$. Âgés de 43 à 56 ans, ils ont entre 13 et 29 ans d'expérience en enseignement au collégial. Ils ont tous bénéficié d'une formation continue (perfectionnement disciplinaire obtenu dans le pays de la langue étrangère enseignée et perfectionnement pédagogique PERFORMA) visant à la fois le développement professionnel et les changements de pratique en lien avec les objectifs des établissements collégiaux.

La collecte des données s'est déroulée au cours des sessions d'été et d'automne 2006. Elle a été informée par la perspective interactionniste symbolique. Cette perspective permet d'examiner le processus d'attribution et de négociation de sens à travers les interactions entre les acteurs et les symboles locaux partagés qui expliquent leur expérience quotidienne (Morrissette, 2010). Elle demande une attention particulière à la culture relationnelle du groupe concerné qui se définit par les formes de communication et l'historique qui régit les interactions entre les membres (Morrissette, 2010). Cette culture induit les symboles observables qui sont créés et communiqués par les acteurs et qui permettent une compréhension et une utilisation partagées pour guider leur action (Sauder, 2005). Autrement dit, ces symboles émergent, se négocient et se transforment au fil du temps au travers des nombreuses interactions entre les membres.

Quant au sens, il se définit en termes d'actions et de conséquences, c'est-à-dire qu'il est attribué à un objet ou à une idée et qu'il est rendu explicite par l'action posée

3. Le groupe est composé de femmes et d'hommes, mais nous n'utilisons que le genre masculin afin de protéger l'anonymat des personnes. 
par un individu ou un groupe (Blumer, 1969). Selon la perspective interactionniste, chaque individu construit différemment un sens à l'égard du monde extérieur selon sa définition de la situation (Thomas, 1923). Le rôle de la situation est donc fondamental pour appréhender l'univers social dans lequel les perceptions subjectives sont projetées dans la réalité et conséquemment matérialisées (Thomas, 1923). Autrement dit, la réalité des acteurs n'est jamais objective. Leur interprétation de la situation influence leur action. Toutefois, tout processus de construction de sens est éminemment social et construit dans les interactions symboliques et la négociation de sens avec d'autres acteurs, que ceux-ci soient présents ou non. Comme le dit Morrissette (2010), «les acteurs agissent en fonction de la façon dont ils définissent les situations, et de l'ajustement de ces définitions à celles des autres» (p. 7).

À la lumière de ces considérations théoriques, nous avons procédé à une analyse de contenu de documents officiels provenant des syndicats, du Conseil supérieur de l'éducation et du ministère de l'Éducation afin de cerner les prescriptions ministérielles jugées problématiques chez les enseignants. Ainsi qu'il a été mentionné précédemment, l'approche-programme, l'approche par compétences, l'évaluation des compétences et l'élaboration de l'épreuve synthèse se sont révélées comme étant les plus complexes. Nous avons réalisé par la suite une ou deux entrevues semi-dirigées de 90 à 180 minutes avec chacun des enseignants participants. Ces entrevues visaient à explorer le sens qu'ils attribuent aux prescriptions ministérielles susmentionnées. Toutefois, les observations entre ces enseignants n'ayant pas été autorisées, notre analyse se limite à la fois aux discours rapportés et à l'analyse de contenu.

L'analyse des données d'entrevues a été effectuée de manière inductive, c'est-àdire par un processus de thématisation consistant à en dégager les idées générales sur le sens attribué aux prescriptions ministérielles, les symboles témoignant d'un changement de conduite chez les enseignants et le type d'interactions sociales qui permettrait une représentation partagée entre les acteurs. Le but de ce processus était de comprendre comment se négocie le sens des changements prescrits dans la pratique enseignante. Dans la section qui suit, nous présentons les résultats de cette analyse qui mettent en lumière la centralité des symboles, le rôle du coordonnateur et celui des interactions sociales dans le processus interprétatif de ces changements.

\section{Résultats}

Dans un premier temps, nous présentons un profil de chacun des participants et dégageons certaines particularités qui leur sont propres. Ainsi, il est possible d'établir des liens entre certaines caractéristiques susceptibles d'expliquer comment chaque individu construit un sens à l'égard du monde extérieur et sa définition de la situation et de ses actions. Dans un second temps, nous présentons les trois dimensions importantes dans la construction et la négociation du sens du changement prescrit dans ce département, que la perspective interactionniste adoptée nous a permis de dégager: 1) l'utilisation d'un symbole pour créer et négocier le sens entre 
les acteurs; 2) les incidences du coordonnateur du département de langues modernes sur la mise en œuvre des changements prescrits et 3) l'importance des interactions sociales.

\section{Benno, le coordonnateur}

Benno est le coordonnateur du département. Il est très impliqué dans la vie institutionnelle du collège. Au début de la réforme, il a participé au comité de rédaction du programme arts et lettres. Aujourd'hui, Benno représente le collège au comité des enseignants du programme arts et lettres et il siège au comité permanent des études préuniversitaires.

Au début du mandat de Benno, son poste de coordonnateur n'a pas été reconnu par l'établissement, puisque chaque département a normalement une seule coordination. Or, il existait deux coordinations au sein du département étudié, scindant le département en deux et engendrant ainsi certaines tensions entre les collègues. Par exemple, un conflit a surgi au sujet du dégrèvement accordé pour la coordination, qui devait être partagé en deux. Benno s'est senti obligé de justifier son poste en travaillant plus d'heures. Il dit s'être distancé de ses collègues par crainte de se faire enlever la coordination. Benno estime avoir eu une attitude défensive envers ses collègues qui se serait manifestée par un comportement de retrait et de repli sur soi. Par ailleurs, Benno juge avoir peut-être pris trop de place par rapport à ses autres collègues afin de défendre la survie des cours d'allemand, lorsqu'il a cru que le collège voulait promouvoir des cours d'espagnol au détriment des cours d'allemand.

Aujourd'hui, son leadership est reconnu à la fois par le collège et ses collègues. Néanmoins, Benno estime que les fonctions de son poste se décrivent majoritairement en termes de tâches administratives supplémentaires par rapport aux autres membres du département. Il dit ne pas avoir un pouvoir décisionnel réel. Selon lui, le pouvoir décisionnel demeure centralisé au niveau de la direction des études.

\section{Les autres participants: Frédéric, Paul et Thomas}

Frédéric est impliqué dans la vie institutionnelle du collège. Il siège au comité Arts et lettres. Il a participé également à l'élaboration de l'épreuve synthèse. Frédéric mentionne sa collaboration à la création d'un double DEC avec Benno. Contrairement à ses deux autres collègues, il s'investit dans la vie institutionnelle. Par ailleurs, il exprime le désir de devenir coordonnateur du département.

Paul n'est pas impliqué dans des comités ou des tables de concertation au sein du collège. Il remarque que les deux collègues siégeant à des comités partagent le même vocabulaire et connaissent davantage les prescriptions ministérielles. Il note également que certaines décisions sont prises à l'extérieur du département à des tables de concertation. Selon Paul, ce sont les personnes du département impliquées dans ces comités qui prennent les décisions et les rapportent dans les réunions départementales. Il ne se sent pas concerné par les changements administratifs.

Thomas donne les cours complémentaires de la formation générale des étu diants au collège. Ses classes sont donc très hétérogènes, puisque les étudiants de tous les programmes préuniversitaires et techniques peuvent s'y inscrire. Il est le seul 
participant qui donne des cours complémentaires. Il se situe un peu à l'écart de ses collègues qui travaillent pour le programme arts et lettres. Thomas affirme vouloir changer sa pratique par sa propre volonté. Il ne le fait pas dans le but de répondre aux exigences du renouveau pédagogique. Il estime qu'un enseignant de langues doit s'informer pour savoir comment changer sa pratique. Ses expériences personnelles de perfectionnement à l'étranger lui ont montré comment il pouvait concrètement modifier sa pratique.

\section{L'épreuve synthèse: symbole conduisant les priorités d'action au sein du département}

Parmi les quatre prescriptions ministérielles analysées, l'épreuve synthèse prédomine dans le discours des enseignants. Tous les participants y ont attribué un sens commun et partagé. Ils définissent l'épreuve synthèse comme étant un projet de fin d'études. Thomas ajoute qu'il s'agit "d'une façon d'obtenir une vue d'ensemble très globale». Il semble y avoir également consensus sur l'utilité de cette épreuve pour démontrer l'acquisition des habiletés langagières, c'est-à-dire la capacité des étudiants à communiquer dans une langue étrangère. Les participants s'entendent également pour dire qu'il s'agit d'un moment clé pour évaluer l'intégration des apprentissages et voir les étudiants performer. Il ressort en outre de l'analyse des données d'entrevues que l'épreuve est devenue un symbole non seulement de qualité du programme et de réussite des étudiants, mais également de compétition et de tension entre les enseignants de différents départements au sein du même programme d'études. Ce symbole exerce une pression constante sur les enseignants de ce département.

\section{[...] il y a une surenchère du projet de fin d'études. C'est devenu un objet de prestige où chaque profil va montrer tout ce qu'il a réussi à faire avec ses étu- diants. (Benno)}

Cette prescription ministérielle exige une réorganisation du travail dans la mesure où la concertation entre enseignants de divers départements au sein d'un même programme d'études est nécessaire pour l'élaborer et la planifier conjointe-

Dans le cas présent, l'épreuve synthèse exerce une forme d'imputabilité interne par le biais du contrôle des résultats de programmes d'études par les enseignants de différentes disciplines. ment. Elle requiert que tous les enseignants concernés dressent un profil de l'étudiant diplômé dans un programme d'études précis et qu'ils définissent la manière dont ils pourront évaluer l'atteinte de ces objectifs (CEEC, 2001). Les défis à relever sont grands pour les enseignants; il s'agit d'une activité collective qui invite des enseignants de différents départements à faire état de la planification de leur enseignement et des moyens privilégiés pour favoriser l'atteinte des compétences. Or, si la cohésion et la collaboration entre les enseignants de différentes disciplines s'opèrent difficilement, il est possible que l'épreuve synthèse ne donne pas les effets escomptés. En examinant la manière dont les enseignants utilisent un symbole au quotidien, il est possible d'en démontrer la force (Sauder, 2005). Dans le cas présent, l'épreuve synthèse exerce une forme d'imputabilité interne par le biais du contrôle des résultats de programmes d'études par les enseignants de différentes disciplines. 
Selon Benno, l'utilisation de l'épreuve synthèse comme objet de prestige est principalement alimentée par les enseignants de différents départements au sein du programme d'études. L'enseignant nous explique que les départements travaillent ensemble pour faire la promotion du programme, car il y a «une perte de clientèle abominable». Benno souligne que le travail entre collègues de différents départements n'est pas toujours évident, parce que certains profils, dont celui de langues modernes, attirent peu d'étudiants et que les collègues d'autres départements se demandent «pourquoi ils tireraient des boulets [...] vous n'attirez pas, pourquoi est-ce nous qui travaillons pour vous? (Benno)

Thomas note que le coordonnateur renforce ce symbole de prestige pour mettre en valeur leur profil.

[...] dans le but de faire une présentation devant un public qui est recherché, les profs d'ici, les profs de là, les profs de l'université, le consulat de l'Allemagne. C'est un vrai show. (Extrait de l'entrevue avec Thomas)

Paul corrobore ces propos en expliquant que l'épreuve synthèse est devenue au cours des années un événement «vraiment officiel». Des invitations sont envoyées par la poste à différents représentants des universités, du consulat et d'écoles secondaires. L'utilisation de ce symbole auprès d'un public cible engendrerait aussi une tension supplémentaire chez les étudiants du programme, selon les participants. En effet, les étudiants finissants doivent composer à la fois avec la pression ressentie devant une épreuve qui détermine l'obtention de leur diplôme et avec celle mise sur eux par le coordonnateur qui tient à ce qu'ils performent bien en public. Thomas nous explique que les étudiants «apprennent par cœur» leurs textes. Les auxiliaires d'enseignement corrigent les textes dans les moindres détails. Les étudiants récitent phrase par phrase tout au long de la session en vue de l'épreuve synthèse.

[...] on ne veut pas se mettre devant les gens, pis montrer que nos étudiants ne savent rien faire, pis qu'ils sont moins bons que ceux de français, pis qu'ils sont moins bons que ceux de cinéma. Alors, il y a une introduction d'une concurrence assez farouche et je me rappelle de scènes où mes étudiants sont sortis de là en pleurant parce que je leur ai dit: Vous n'allez pas me faire honte. [...] Vous représentez le programme, vous représentez le collège. C'est correct, moi je ne te laisse pas produire [n'importe quoi]. Ça, ç'a jamais existé avant. (Benno)

L'origine de ce symbole n'est toutefois pas claire. Il est difficile, à partir des témoignages recueillis, de déduire si la considération de l'épreuve synthèse comme objet de prestige est suscitée soit par la direction des études du collège, soit par les collègues des autres départements ou par l'interprétation du coordonnateur luimême. Néanmoins, ce qui est apparent, c'est que la compétition et la tension sont entretenues par le coordonnateur - une façon de justifier l'existence du programme. La survie du programme et la perte de clientèle préoccupent tous les participants. Le fait que chacun travaille à la survie du programme permet donc au coordonnateur de légitimer les actions à l'égard de l'épreuve synthèse. 


\section{Les incidences du coordonnateur sur la mise en œuvre des changements au sein du département}

Le leader joue un rôle lorsqu'il est question de construction et négociation du sens (Coburn, 2001; Desimone, 2002; Hargreaves, 1991). Ce rôle est de construire un sens par rapport à l'environnement interne et externe de l'organisation pour ensuite entreprendre les changements souhaités (Gioia et Chittipeddi, 1991). Le sens que le leader attribue à son rôle, à une idée ou à la profession façonnera la manière dont les enseignants construiront du sens par la suite et l'ajustement qu'ils feront à celui des autres. Dans le cas présent, Benno décrit son rôle de leader comme celui d'un pair parmi ses pairs. Il ajoute que son «leadership c'est d'essayer que les gens soient bien dans leur travail, pis d'encourager la qualité du programme». Benno a construit un sens très fort à l'égard du programme. Il s'y réfère en termes de "gagne-pain». Le programme revient à maintes reprises dans son discours comme étant sa priorité d'action. De plus, Benno se dit mal à l'aise en présence de conflits, mais explique que l'importance qu'il accorde au programme lui a permis d'y faire face:

[...] ce que je ne ferai pas pour moi, je vais le faire pour le programme. Mais c'était pour le programme, ce n'était pas pour moi. Alors, pour le programme, on trouve beaucoup d'énergie, j'ai beaucoup de force, c'est ben important ce programme-là. C'est ça. Ça ne me surprendrait pas que tu retrouves des réactions semblables chez mes collègues aussi. On y tient beaucoup à notre programme. (Benno)

Toutefois, Benno définit la situation en termes de réussite du programme et semble prioriser davantage les discussions entre enseignants autour de ce thème. Il se distancie un peu plus de la pratique enseignante et considère que cette pratique est isolée et individuelle. Selon lui, il est possible de créer un lieu où les enseignants abordent un problème afin que quelqu'un d'autre puisse le résoudre. Les enseignants sont donc conviés à se référer à une personne possédant les compétences et les outils pour résoudre les problèmes qu'ils pourraient rencontrer dans leur pratique. Selon Coburn (2001), les leaders en position d'autorité créent les conditions nécessaires qui favoriseront les échanges entre les enseignants, encourageant ainsi la représentation partagée au sein d'un groupe. Dans le cas présent, le coordonnateur semble créer des conditions où le partage des représentations porte davantage sur le programme.

De plus, le coordonnateur estime avoir eu une attitude défensive envers ses collègues qui se serait manifestée par une conduite de retrait et de repli sur soi. La définition de son rôle de leader semble donc avoir une incidence sur la manière dont il gère les relations avec les autres enseignants du département. Contrairement aux trois autres participants, les interactions ne sont pas très importantes pour lui et ressortent très peu dans son discours. Selon Benno:

On a trouvé des façons de fonctionner qui permettent le respect. Je pense que les gens se respectent. Je ne pense pas que ça soit l'amitié délirante, mais ce n'est pas le but de l'affaire-là [...] pour moi, c'est devenu du travail, c'est du travail, pis la vie privée, c'est la vie privée. Ça, je le maintiens beaucoup. 
Peut-être, je pense que les collègues aimeraient ça des fois qu'il y ait plus (ricanement) de chaleur [...] mais ça ne marche pas. (Benno)

Considérant que les effets du leadership peuvent se faire ressentir sur les relations de travail collaboratives (Hargreaves, 1991), le retrait exprimé par le coordonnateur par rapport à ses collègues et le sens donné à la pratique enseignante comme étant «un métier isolé» semblent avoir eu des incidences sur le manque de représentation partagée entre ces enseignants en ce qui a trait aux changements dans la pratique. Or, Desimone (2002) précise que la participation du leader est un des plus importants prédicteurs de changement de pratique. En effet, les discussions concernant la pratique enseignante, les problèmes rencontrés en classe ou l'innovation pédagogique ne semblant pas faire partie des priorités du coordonnateur, la construction et la négociation de sens du changement prescrit demeurent davantage d'ordre individuel. Les changements de pratique semblent donc émerger davantage d'un intérêt ou d'un désir d'améliorer la pratique plutôt que d'une représentation partagée d'une prescription ministérielle.

\section{Incidences des interactions sociales sur la mise en œuvre des changements dans la pratique pédagogique}

Les interactions sociales jouent un rôle significatif dans la construction de sens dans et par le collectif. Certains auteurs ont établi un lien entre le succès du changement entrepris et la qualité des interactions sociales chez les enseignants (Fullan, 2001; Hargreaves, 1991; Spillane et al., 2002). À titre d'exemple, l'influence d'une culture de collaboration augmenterait la participation et l'engagement des enseignants et permettrait l'accès à de nouvelles idées et connaissances (Day et al., 2005). Dans le cas présent, il semble y avoir très peu de collaboration entre les membres de ce département pour ce qui regarde la pratique pédagogique. Frédéric, entre autres, en témoigne:

J'aurais aimé ça avoir collaboré beaucoup plus. Je trouve qu'on s'entend bien sur un plan personnel. Ce qui est drôle parce que j'aurais pensé le contraire. On se soutient si y en a qui a un problème [...] Mais côté [...] travail chacun fait vraiment sa petite affaire. Y'en pas de collaboration, y'en a vraiment pas. (Frédéric)

Ainsi que le relèvent Desimone (2002), Schmidt et Datnow (2005), de même que Zembylas $(2002,2005)$, les relations de pouvoir, les tensions et les conflits semblent avoir un impact considérable sur les conduites adoptées par les enseignants lorsqu'il est question de changement. Dans le cas présent, il semble qu'ils centrent les interactions sur des thèmes d'ordre administratif et excluent la collaboration concernant la mise en œuvre de l'approche par compétences et son évaluation. Ce faisant, la négociation de sens se jouerait davantage autour de l'épreuve synthèse. Il semble y avoir très peu d'incidences de l'approche par compétences et des nouvelles exigences en matière d'évaluation des apprentissages sur la pratique enseignante. Nous notons, entre autres, les propos de Thomas qui expriment une certaine tension dans les interactions sociales entre les enseignants. Il les décrit comme étant froides, distantes et difficiles. 
Quand c'est d'une façon individuelle, c'est très bien, très humain, très chaleureux. Mais quand on est en équipe, on dirait que chaque pion prend sa place. Alors, ça devient froid, ça devient distant, ça devient ... [silence] quelquefois ça devient difficile [silence]. Je les trouve difficiles [petit ricanement]. (Thomas)

Coburn (2001) estime que les conversations dans les cadres formels et informels peuvent jouer un rôle déterminant dans la construction et la négociation de sens à l'égard des changements prescrits dans la pratique enseignante. Toutefois, il semble que les courtes périodes d'échanges, les divergences d'opinions et de sens à l'égard de la pratique enseignante causant parfois des problèmes de communication, ne permettent pas aux enseignants de s'engager en profondeur dans les changements souhaités. De plus, selon les propos de Thomas et Frédéric, l'historique des interactions sociales, parfois distantes, tendues et difficiles, pourrait être également à l'origine de certaines représentations non partagées et d'une absence de négociation de sens entre les enseignants concernant la pratique.

Paul, quant à lui, est plus positif que ses autres collègues en ce qui a trait aux interactions sociales au sein du département. Tout comme les trois autres participants, il les qualifie de respectueuses.

Tout le monde se respecte. Le premier mot que je dirais, c'est respect. Et il y a beaucoup d'écoute, en général. Normalement, il n'y a aucune décision qui est prise unilatéralement. On essaie toujours d'en discuter. Ça arrive qu'il y ait des choses qui soient décidées, pis on en discute après. Ça, ça me titille un peu, je dirais. J'aime bien donner mon opinion sur ce qui se passe. Mais, c'est rare. (Paul)

Contrairement au coordonnateur, les trois enseignants expriment le désir de collaborer avec leurs collègues concernant la pratique enseignante. Il serait hâtif d'affirmer que l'historique de leurs interactions soit à l'origine du manque de collaboration. Nous constatons toutefois que les efforts du coordonnateur en lien avec le programme l'éloignent davantage des préoccupations concernant la pratique enseignante.

\section{Conclusion}

Les constats tirés de cette étude de cas tendent à mettre en lumière l'importance du sens donné aux changements par les leaders et les autres acteurs, de même que des symboles comme l'épreuve synthèse conduisant la gestion et l'appropriation locales des changements. Ces symboles peuvent affecter les interactions sociales au sein d'un groupe, voire modifier les intentions d'une prescription ministérielle (Sauder, 2005). Dans le cas présent, l'interprétation de l'épreuve synthèse comme objet de prestige explique l'insistance répétée du coordonnateur à mettre en place des mécanismes qui assurent la «performance publique» des étudiants. Ce symbole 
incarne non seulement les expériences quotidiennes de ces enseignants, il les façonne également (Sauder, 2005).

Les constats réaffirment aussi l'importance du coordonnateur dans la construction et la négociation du sens pour effectuer des changements de pratique prescrits (Coburn, 2001; Hargreaves, 1991; Spillane et al., 2002). En effet, le coordonnateur ne donne pas aux enseignants la possibilité d'échanger entre eux au sujet des changements à apporter dans leur pratique. Dans une perspective interactionniste, les actions, les constats et la définition d'un rôle sont tous empreints d'un sens attribué par l'individu et qui influence la manière dont celui-ci les interprète. Or, la définition de la situation par Benno, c'est-à-dire son retrait par rapport à ses collègues, son interprétation de la pratique enseignante comme une activité solitaire et son rôle en tant que leader administratif et non pédagogique, a pour conséquence une orientation axée sur les aspects administratifs du programme et très peu sur la pratique enseignante. De plus, la dynamique des interactions sociales au sein de ce département paraît défavorable aux échanges entre collègues. Le climat ne semble pas propice à la création du lien de confiance nécessaire, puisque les priorités du coordonnateur sont concentrées principalement sur le programme, y compris l'épreuve synthèse.

Comme le mentionnent Weitz et Rosenbaum (2008), les symboles peuvent émerger au sein d'un groupe lorsque des incitations ou des menaces y sont exercées, menant à un changement de conduites parfois inattendu chez les individus. Dans cette perspective, notre analyse pourrait être complétée par un examen de l'influence des autres acteurs de l'établissement qui auraient pu contribuer à l'émergence des symboles définissant les priorités d'action dans une période où des changements de pratiques sont souhaités. Il serait par exemple intéressant d'examiner quel a été le rôle de la direction des études ou des autres coordonnateurs dans l'émergence du symbole de l'épreuve synthèse comme objet de prestige.

\section{Références bibliographiques}

CENTRALE DES SYNDICATS DU QUÉBEC (1999). La formation du personnel enseignant des collèges. Avis au Conseil supérieur de l'éducation par la Fédération des enseignantes et enseignants de cégep (FEC).

COBURN, C. E. (2001). Collective sensemaking about reading: How teachers mediate reading policy in their professional communities. Educational Evaluation and Policy Analysis, 23(2), 145-170.

COMITÉ PATRONAL DE NÉGOCIATION DES COLLÈGES (CPNC) (2004). Dépôt patronal en vue du renouvellement de la convention collective liant la FNEEQ (CSN) et le CPNC. 
CONSEIL SUPÉRIEUR DE L'ÉDUCATION (1998). Enseigner au collégial: une pratique professionnelle en renouvellement. Québec: Gouvernement du Québec, 3(1).

CONSEIL SUPÉRIEUR DE L'ÉDUCATION (2000). La formation du personnel enseignant du collégial : un projet collectif enraciné dans le milieu. Avis au ministère de l'Éducation, Québec, 5(2).

CONSEIL SUPÉRIEUR DE L'ÉDUCATION (2003). L'appropriation locale de la réforme: un défi à la mesure de l'école secondaire. Avis au ministère de l'Éducation, Québec, 29 p.

DESIMONE, L. (2002). How can comprehensive school reform models be successfully implemented? Review of Educational Research, 72(3), 433-479.

DORAIS, S. (1992). Dossier: Pour l'animation et le perfectionnement dans une perspective d'approche-programme. Module 2. Dans une perspective d'approche-programme... des concepts et des conceptions, Sherbrooke: PERFORMA, juin.

FULLAN, M. (2001). The New Meaning of Educational Change. New York: College Press.

GINGRAS, P.-É. (1992a). Les cégeps d'hier à demain. 1. Vers la réforme scolaire. Pédagogie collégiale, 6(1), 4-7.

GINGRAS, P.-É. (1992b). Les cégeps d'hier à demain. 2. L'euphorie des bâtisseurs. Pédagogie collégiale, 6(2), 4-7.

GIOIA, D. et CHITTIPEDDI, K. (1991). Sensemaking and sensegiving in strategic change initiation. Strategic Management Journal, 12(6), 433-448.

GOULET, J.-P. (1990). L’approche-programme : quelques changements en perspective. Pédagogie collégiale, 4(2), 6-8.

HARGREAVES, A. (1991). Contrived collegiality. The micropolitics of teacher collaboration. Dans J. Blase (dir.), The Politics of Life in Schools: Power, Conflict and Cooperation. Newbury Park, CA: Sage Publications: 46-71.

KARSENTI, T. et DEMERS, S. (2000). L'étude de cas. Dans T. Karsenti et L. SavoieZacj (dir.). Introduction à la recherche en éducation (p. 225-248). Sherbrooke : Éditions du CRP.

MERRIAM, S. B. (1988). Case Study Research in Education. San Francisco : JosseyBass Publishers.

MINISTÈRE DE L'ENSEIGNEMENT SUPÉRIEUR ET DE LA SCIENCE (1992). Des collèges pour le Québec du XXI siècle: les orientations d'avenir et mesures de renouveau. Québec, $39 \mathrm{p}$.

MORRISSETTE, J. (2010). Une perspective interactionniste: un autre point de vue sur l'évaluation des apprentissages. SociologieS. [En ligne] http://sociologies.revues.org/3028?\&id=3028. 
QUIVY, R. et VAN CAMPENHOUDT, L. (1988). Manuel de recherche en sciences sociales. Paris: Dunod.

ROBERT, M. et TONDREAU, J. (1997). L'école québécoise. Débats, enjeux et pratiques sociales. Une analyse de l'éducation pour la formation des maîtres. Montréal:

CEC.

SAUDER, M. (2005). Symbols and contexts: An interactionist approach to the study of social status. The Sociological Quarterly, 46, 279-298.

SCHMIDT, M. et DATNOW, A. (2005). Teachers' sense-making about comprehensive school reform: The influence of emotions. Teaching and Teacher Education, 21(5), 949-965.

SPILLANE, J., REISER, B. et al. (2002). Policy implementation and cognition: Reframing and refocusing implementation research. Review of Educational Research, 72(3), 387-431.

THOMAS, W. I. (1923). The Unadjusted Girl. Boston: Brown \& Co.

VAN DER MAREN, J.-M. (1996). Méthodes de recherche pour l'éducation (2e éd.). Montréal et Bruxelles: PUM et de Boeck.

WEITZ-WHITE, K. et ROSENBAUM, J. E. (2008). Inside the black box of accountability: How high-stakes accountability alters school culture and the classification and treatment of students and teachers. Dans A. Sadovnik, J. A. O’Day, G. W. Bohrnstedt et K. M. Borman (dir.), No Child Left Behind and the Reduction of the Achievement Gap (p. 97-114). New York: Routledge Taylor \& Francis Group.

ZEMBYLAS, M. (2002). Constructing genealogies of teachers' emotions in science teaching. Journal of Research in Science Teaching, 39(1), 79-103. 